\title{
DETERMINANT OF THE NEUMANN OPERATOR ON SMOOTH JORDAN CURVES
}

\author{
JULIAN EDWARD AND SIYE WU \\ (Communicated by Barbara L. Keyfitz)
}

\begin{abstract}
Using the method of heat kernel expansion, the determinant of the Neumann operator on an arbitrary smooth Jordan curve is shown to be equal to the circumference.
\end{abstract}

\section{INTRODUCTION}

The definition of determinants of Laplacians in terms of the zeta function comes up in the study of analytic torsion [5,6]. It was soon discovered that the zeta function actually provides a technique of regularizing quadratic path integrals in quantum field theories [3]. Recently, this subject has been of considerable interest in mathematical physics. For instance, the determinants of differential operators such as the Laplacians on a compact Riemann surface were calculated in the first quantized string theory [2]. These determinants are also related to the inverse spectral problems of the operators [4, 9]. On the other hand, the zeta function regularization of the determinant depends only on the spectrum of the operator in question; the operator could be a differential operator, a pseudodifferential operator, or any other semipositive self-adjoint operator acting on a Hilbert space whose heat operator is of trace class. In practice, evaluation of the determinants of operators of more general type may be useful in the study of effective quantum field theories, where the action need not be local.

In this paper, we calculate the determinant of the Neumann operator on an arbitrary smooth Jordan curve. The paper is organized as follows: $\S 2$ is a review of the general properties of the Neumann operator. In particular, there is a simple relation between Neumann operators of two planar regions related by a conformal map. In $\S 3$, we study the heat kernel expansion and the zeta function of the Neumann operator. In $\S 4$, we calculate the determinant. The

Received by the editors February 16, 1989 and, in revised form, January 2, 1990.

1980 Mathematics Subject Classification (1985 Revision). Primary 35D05, 58 G25.

Key words and phrases. Zeta function, determinant, heat kernel.

The first author was supported in part by a research assistantship at MIT. The second author was supported in part by a Wang Fellowhip. 
functional variation of the determinant is related to the behavior of the heat kernel over small time.

\section{THE NEUMANN OPERATOR}

Let $\Omega$ be a simply connected bounded region with a smooth boundary in a finite-dimensional Euclidean space. The Neumann operator is a linear operator acting on the space of functions on its boundary $\partial \Omega$. A function $\varphi$ on $\partial \Omega$ has a unique harmonic extension $\tilde{\varphi}$ on $\Omega$ such that

$$
\left\{\begin{array}{l}
\Delta \tilde{\varphi}=0 \text { in } \Omega \\
\left.\tilde{\varphi}\right|_{\partial \Omega}=\varphi
\end{array}\right.
$$

The Neumann operator $N_{\partial \Omega}$ is defined by

$$
N_{\partial \Omega} \varphi=\left.\frac{\partial \tilde{\varphi}}{\partial n}\right|_{\partial \Omega} .
$$

More precisely, $N_{\partial \Omega}$ is the closure of the above linear operation in $L^{2}(\partial \Omega)$. It is a first order elliptic pseudodifferential operator.

For simplicity, we use the notation $\varphi$ for $\tilde{\varphi}$ unless confusion might result. Consider the following three equalities derived from Green's theorem:

$$
\begin{gathered}
\int_{\partial \Omega} \bar{\varphi}_{1} \frac{\partial}{\partial n} \varphi_{2}-\varphi_{2} \frac{\partial}{\partial n} \bar{\varphi}_{1}=\int_{\Omega} \bar{\varphi}_{1} \Delta \varphi_{2}-\varphi_{2} \Delta \partial \bar{\varphi}_{1} \\
\int_{\partial \Omega} \bar{\varphi} \frac{\partial}{\partial n} \varphi=\int_{\Omega} \bar{\varphi} \Delta \varphi+|\nabla \varphi|^{2} \\
\int_{\partial n} \frac{\partial}{\partial n} \varphi=\int_{\Omega} \Delta \varphi
\end{gathered}
$$

Equation (2.3) implies that $N_{\partial \Omega}$ is selfadjoint; (2.4) implies that $N_{\partial \Omega}$ is semipositive and definite, the kernel being the space of constant functions on $\partial \Omega$, so that the dimensions of its kernel and cokernel are both $1 ;(2.5)$ implies that the image of $N_{\partial \Omega}$ is the set of functions whose integrals over $\partial \Omega$ are zero.

Because $N_{\partial \Omega}$ is an elliptic and selfadjoint operator on a compact space $\partial \Omega$, its spectrum is real and discrete. As an example, if $\Omega$ is the unit disk $D=\{z \in$ $\mathbb{C}:|z|<1\}$, then $\partial D$ is the unit circle $S^{1}$. The functions $\varphi_{n}(\theta)=e^{i n \theta} / \sqrt{2 \pi}$, $0 \leq \theta<2 \pi, n \in \mathbb{Z}$ are eigenfunctions of $N_{\partial D}$ with corresponding eigenvalues $|n|$. Therefore, the spectrum of $N_{\partial D}$ is the set of nonnegative integers, each nonzero eigenvalue having multiplicity 2 .

In two dimensions, any simply connected bounded region $\Omega$ can be related to the unit disk via a conformal map $f: D \rightarrow \Omega$ such that when restricted to the boundary $\partial D, f$ is a diffeomorphism onto $\partial \Omega$. The pull-back of the metric on $\partial \Omega$ to $\partial D$ is $d s^{2}=d \theta^{2} / g(\theta)^{2}$, where

$$
g(\theta)=\frac{1}{\left|f^{\prime}\left(e^{i \theta}\right)\right|}, \quad 0 \leq \theta<2 \pi
$$


is a positive function on $\partial D$ determined by $f$. In particular, the circumference of $\partial \Omega$ is

$$
C[\partial \Omega]=\int_{0}^{2 \pi} \frac{1}{g(\theta)} d \theta .
$$

The induced map $f^{*}: C^{\infty}(\partial \Omega) \rightarrow C^{\infty}(\partial D)$ is not in general an isometry under the standard norms of the two Hilbert spaces $L^{2}(\partial \Omega)$ and $L^{2}(\partial D)$. Instead, the induced inner product on $L^{2}(\partial D)$ via $f^{*}$ is

$$
\left\langle\varphi_{1}, \varphi_{2}\right\rangle_{f}=\int_{0}^{2 \pi} \frac{1}{g(\theta)} \bar{\varphi}_{1}(\theta) \varphi_{2}(\theta) d \theta
$$

If $\varphi$ is a harmonic function on $\Omega$, then $f^{*} \varphi=\varphi \circ f$ is harmonic on $D$, because $f$ is conformal. Since the push-forward of the outer normal vector is

$$
f_{*}\left(\left.\frac{\partial}{\partial n}\right|_{\partial D}\right)=\left.\frac{1}{g(\theta)} \frac{\partial}{\partial n}\right|_{\partial \Omega},
$$

the pull-back of the Neumann operator is given by

$$
f^{*} N_{\partial \Omega} f^{*-1}=g N_{\partial D} .
$$

Notice that it is self-adjoint only under the induced norm given by $(2.8)$.

\section{THE HEAT KERNEL EXPANSION}

Let $A$ be a semipositive self-adjoint elliptic pseudodifferential operator on a compact manifold $M$ and $\left\{\varphi_{i}\right\}$ be a complete set of normalized eigenfunctions of $A$ with corresponding eigenvalues $\lambda_{i}$. The heat kernel of the operator $A$ restricted to the diagonal

$$
H_{t}(x, x)=\sum e^{-t \lambda_{i}}\left|\varphi_{i}(x)\right|^{2}, \quad x \in M, t>0
$$

has an asymptotic expansion at small $t$

$$
H_{t}(x, x) \sim \sum_{n=-d}^{\infty} a_{n}(x, x) t^{n / m}, \quad \text { as } t \rightarrow 0^{+},
$$

where $m$ is the order of $A$ and $d$ is the dimension of $M$. The purpose of this section is to compute $a_{0}(x, x)$ for the Neumann operator $N_{\partial \Omega}$ on a smooth Jordan curve $\partial \Omega$.

Note that $a_{0}(x, x)$ is not changed if we replace $N_{\partial \Omega}$ by $A=N_{\partial \Omega}+P$, where $P$ is the projection operator onto the kernel of $N_{\partial \Omega}$. The latter has the advantage that its spectrum consists of positive real numbers only. For such an operator, we can define its complex power $A^{s}$, and the Schwartz kernel restricted to the diagonal is

$$
K_{s}(x, x)=\sum \lambda_{i}^{s}\left|\varphi_{i}(x)\right|^{2} .
$$


The sum converges absolutely when $\operatorname{Re} s<-d / m$ and extends meromorphically in $s$ to the complex plane in such a way that it is regular at $s=0$ [8]. It is well known that

$$
K_{s}(x, x)=\frac{1}{\Gamma(s)} \int_{0}^{\infty} t^{s-1} H_{t}(x, x) d t ;
$$

i.e., $K_{s}(x, x) \Gamma(s)$ is the Mellin transform of $H_{t}(x, x)$. Taking the inverse transform, we get

$$
a_{0}(x, x)=K_{0}(x, x) .
$$

Since $A$ is a first-order operator and the ray of minimal growth can be chosen as the negative real axis, the Schwartz kernel at $s=0$ is, in [8],

$$
K_{0}(x, x)=\frac{1}{2 \pi} \int_{|\xi|=1} d \xi \int_{0}^{\infty} d t b_{-2}(x, \xi,-t),
$$

where

$$
\sigma\left(B_{\lambda}\right)(x, \xi)=\sum_{j=0}^{\infty} b_{-1-j}(x, \xi, \lambda)
$$

is the total symbol of the parametrix $B_{\lambda}$ of $A-\lambda I$. Since the total symbol of $A$ is $\sigma(A)(x, \xi)=|\xi|$ if $x$ is the arclength parameter, we have

$$
\sigma\left(B_{\lambda}\right)(x, \xi)=\frac{1}{|\xi|-\lambda},
$$

which implies that

$$
b_{-1-j}(x, \xi, \lambda)= \begin{cases}\frac{1}{|\xi|-\lambda}, & j=0, \\ 0, & j>0 .\end{cases}
$$

This shows that $K_{0}(x, x)=0$, or equivalently,

$$
a_{0}(x, x)=0
$$

for the Neumann operator $N_{\partial \Omega}$.

\section{Calculation of the determinant}

Recall that the zeta function of a semipositive selfadjoint elliptic pseudodifferential operator $A$ is defined by

$$
\zeta_{A}(s)=\operatorname{tr}^{\prime} A^{-s}=\sum^{\prime} \frac{1}{\lambda_{i}^{s}},
$$

where prime means summing over the nonzero eigenvalues $\lambda_{i} \neq 0$ only. The zetafunction regularization of the determinant is, in [3],

$$
\operatorname{det}^{\prime} A=e^{-\zeta_{A}^{\prime}(0)} \text {. }
$$

As a consequence,

$$
\operatorname{det}^{\prime}(c A)=c^{\zeta_{A}(0)} \operatorname{det}^{\prime} A .
$$


If $A$ is an elliptic operator of strictly positive spectrum on a compact manifold $M$, then

$$
\zeta_{A}(s)=\int_{M} d v_{x} K_{-s}(x, x),
$$

where $d v$ is the volume form on $M$. (3.5) implies that

$$
\zeta_{A}(0)=a_{0},
$$

where

$$
a_{n}=\int_{M} d v_{x} a_{n}(x, x)
$$

are the coefficients in the heat kernel expansion

$$
\operatorname{tr} e^{-t A}=\int_{M} d v_{x} H_{t}(x, x) \sim \sum_{n=-d}^{\infty} a_{n} t^{\frac{n}{m}}, \text { as } t \rightarrow 0^{+} .
$$

In general, if the kernel of $A$ is nonzero, we have

$$
\zeta_{A}(0)=a_{0}-\operatorname{dim} \operatorname{ker} A .
$$

For example, (3.10) implies

$$
\zeta_{N_{\partial \Omega}}(0)=-1,
$$

which does not depend on the shape of the region $\Omega$.

The zeta function of the Neumann operator $N_{\partial D}$ on the unit circle is

$$
\zeta_{N_{\partial D}}(s)=\sum_{n=1}^{\infty} \frac{2}{n^{s}}=2 \zeta(s),
$$

where $\zeta(s)$ is the Riemann zeta function satisfying $\zeta(0)=-1 / 2$ and $\zeta^{\prime}(0)=$ $-(1 / 2) \log 2 \pi$. This agrees with (4.9). Moreover, the determinant is

$$
\operatorname{det}^{\prime} N_{\partial D}=e^{-2 \zeta^{\prime}(0)}=2 \pi .
$$

In general, the eigenvalue problem is not exactly soluble, but the determinant can be expressed by the heat kernel [1,7]:

$$
\begin{gathered}
\log \operatorname{det}^{\prime} A=\text { coefficient of } \varepsilon^{0} \text { in the asymptotic expansion of } \\
\qquad-\int_{\varepsilon}^{\infty} \frac{d t}{t} \operatorname{tr}^{\prime} e^{-t A}, \quad \text { as } \varepsilon \rightarrow 0^{+} .
\end{gathered}
$$

If $A$ is an operator like $N_{\partial \Omega}=g f^{*-1} N_{\partial D} f^{*}$, where for simplicity we used the same notation $g$ for $g \circ f^{-1}$, its determinant can be calculated by the method of functional variation, i.e., by studying how the determinant changes under a small variation $\delta g$. The eigenvalues and eigenvectors of $N_{\partial \Omega}$ are not always fixed under such variations, but the kernel consists of constant functions only. Since

$$
\delta N_{\partial \Omega}=\delta g f^{*-1} N_{\partial D} f^{*}=\delta \log g N_{\partial \Omega},
$$


we have

$$
\begin{aligned}
\delta\left[-\int_{\varepsilon}^{\infty} \frac{d t}{t} \operatorname{tr}^{\prime} e^{-t N_{\partial \Omega}}\right] & =-\int_{\varepsilon}^{\infty} \frac{d t}{t} \operatorname{tr}\left(-t \delta N_{\partial \Omega} e^{-t N_{\partial \Omega}}\right) \\
& =\operatorname{tr}\left[\delta \log g \int_{\varepsilon}^{\infty} N_{\partial \Omega} e^{-t N_{\partial \Omega}} d t\right] \\
& =\operatorname{tr}\left[\delta \log g\left(e^{-\varepsilon N_{\partial \Omega}}-P\right)\right]
\end{aligned}
$$

where $P$ is the projection onto the kernel of $N_{\partial \Omega}$, as in $\S 3$. From the heat kernel expansion (3.2), we get

$$
\begin{aligned}
\delta \log \operatorname{det}^{\prime} N_{\partial \Omega} & =\text { coefficient of } \varepsilon^{0} \text { in } \operatorname{tr}\left[\delta \log g\left(e^{-\varepsilon N_{\partial \Omega}}-P\right)\right] \\
& =\int_{\partial \Omega} d v_{x} \delta \log g(x)\left[a_{0}(x, x)-\left|\varphi_{0}(x)\right|^{2}\right],
\end{aligned}
$$

where $\varphi_{0}$ is the normalized function in $\operatorname{ker} N_{\partial \Omega}$, and $a_{0}(x, x)$ vanishes identically from (3.10). If we pull the integral back to $\partial D$, then since $f^{*} d v_{x}=$ $g(\theta)^{-1} d \theta$ and $f^{*} \varphi_{0}(\theta)=C[\partial \Omega]^{-1 / 2}$, we get

$$
\begin{aligned}
\delta \log \operatorname{det}^{\prime} N_{\partial \Omega} & =-\int_{0}^{2 \pi} \frac{d \theta}{g(\theta)} \delta \log g(\theta) \frac{1}{C[\partial \Omega]} \\
& =\frac{1}{C[\partial \Omega]} \int_{0}^{2 \pi} d \theta \delta \frac{1}{g(\theta)} \\
& =\delta \log C[\partial \Omega] .
\end{aligned}
$$

The determinant is then evaluated by solving this functional differential equation. When $\Omega=D$, $\operatorname{det}^{\prime} N_{\partial D}=2 \pi=C[\partial D]$. Hence, for an arbitrary planar region $\Omega$,

$$
\operatorname{det}^{\prime} N_{\partial \Omega}=C[\partial \Omega] \text {. }
$$

In particular, if $\Omega$ is a disk of radius $r$, then

$$
\operatorname{det}^{\prime} N_{\partial \Omega}=2 \pi r \text {. }
$$

This agrees with (4.3), since $N_{\partial \Omega}=r^{-1} N_{\partial D}$.

\section{ACKNOWLEDGMENTS}

The authors wish to thank R. Melrose for suggesting the problem and the referee for useful comments.

\section{REFERENCES}

1. J. Cheeger, Analytic torsion and the heat equation, Ann. of Math. 109 (1979), 259-322.

2. E. D'Hoker and D. H. Phong, On determinants of Laplacians on Riemann surfaces, Commun. Math. Phys. 104 (1986), 537-545.

3. S. W. Hawking, Zeta function regularization of path integrals in curved spacetime, Commun. Math. Phys. 55 (1977), 133-148.

4. B. Osgood, R. Phillips, and P. Sarnak, Extremals of determinants of Laplacians, J. Funct. Anal. 80 (1988), 148-211. 
5. D. B. Ray and I. M. Singer, R-torsion and the Laplacian on Riemannian manifolds, Adv. in Math. 7 (1971), 145-210.

6. __ Analytic torsion for complex manifolds, Ann. of Math. 98 (1973), 154-177.

7. S. Rosenberg, The variation of the de Rham zeta function, Trans. Amer. Math. Soc. 299 (1987), 535-557.

8. R. T. Seeley, Complex powers of an elliptic operator, Amer. Math. Soc. Proc. Symp. Pure Math. 10 (1967), 288-307.

9. S. A. Wolpert, Asymptotics of the spectrum and the Selberg zeta function on the space of Riemann surfaces, Commun. Math. Phys. 112 (1987), 283-315.

Department of Mathematics, University of Toronto, Toronto, Ontario M5S 1A1 CANADA

Department of Mathematics, Massachusetts institute of Technology, Cambridge, MASSACHUSETTS 02139 